\title{
PHAGE TYPING AND MULTIDRUG RESISTANCE PROFILE IN $S$. TYPHIMURIUM ISOLATED FROM DIFFERENT SOURCES IN BRAZIL FROM 1999 TO 2004
}

\author{
Christiane Soares Pereira $^{1 *}$; Luciane Martins Medeiros ${ }^{1}$; Renata Garcia Costa ${ }^{1}$; Márcia Lima Festivo ${ }^{1}$; \\ Eliane Moura Falavina dos Reis ${ }^{1}$; Liliane Miyuki Seki ${ }^{1}$; Dália dos Prazeres Rodrigues ${ }^{1}$
}

${ }^{1}$ Laboratório de Referência Nacional de Cólera e Enteroinfecções Bacterianas, Laboratório de Enterobactérias, Departamento de Bacteriologia, Instituto Oswaldo Cruz, Fundação Instituto Oswaldo Cruz, Rio de Janeiro, RJ, Brasil

Submitted: August 31, 2006; Returned to authors for corrections: November 13, 2006; Approved: March 19, 2007.

\begin{abstract}
Salmonella Typhimurium has become a widespread cause of salmonellosis among humans and animals worldwide. In Brazil, Salmonella Typhimurium (STM) is one of the most prevalent serovars isolated from food for human consumption. The uncontrolled sale and use of antimicrobials in agriculture and for treating human patients contributes to increase multidrug resistance of this serovar. In the present study, a total of 278 STM isolates from different sources and regions of Brazil over the period 1999 to 2004 were phage typed and analyzed for their antimicrobial resistance profile at Laboratory of Enterobacteria, Oswaldo Cruz Institute, FIOCRUZ. The main STM phage types isolated were DT 193 (64.3\%), DT $19(17.4 \%)$ and DT $18(4 \%)$. Others phage types as DT $10(2 \%)$, DT $27(3.24 \%)$, DT $13(0.36 \%)$, DT $22(0.36 \%)$, DT 28 $(0.36 \%)$, DT $29(0.36 \%)$ and DT $149(0.36 \%)$ were obtained in low percentages. A total of 54\% STM strains were resistant to three or more antimicrobial classes, while no resistance to third generation cephalosporin or ciprofloxacin was identified in these strains. Those results show the STM phage types circulating among animals, food for human consumption and humans in Brazil as well as the increasing of multidrug resistance. The surveillance of STM strains based on phage typing and antimicrobial resistance profile are useful for detecting outbreaks, identifying sources of infection and implementing prevention and control measures.
\end{abstract}

Key-words: Salmonella Typhimurium, Phage Types, Multidrug resistance

\section{INTRODUCTION}

Gastroenteritis associated with Salmonella enterica is an important foodborne disease throughout the world. More than 2500 Salmonella serovars are recognized and most of them are capable of infecting a variety of animal species, including humans (6).

In Brazil, Salmonella Typhimurium (STM) is the most prevalent serotype isolated from animals (especially swine) and food for human consumption. An important characteristic of this serovar is the emergence of multidrug resistant phenotypes. The rapid spread of multidrug resistance is a result of many factors such as the uncontrolled sale and extensive use of antimicrobials in agriculture and for treating patients. This phenomenon may be complex especially when bad hygiene is applied at rendering animal to human consumption and also when there is mishandling of food at home (7).

Phage typing has enabled differentiation of Salmonella Typhimurium into more than 200 definitive phage types (DTs). Some of these phage types appear with high prevalence in certain geographical areas. Phage type DT 104, one of the most common multidrug resistant, is pandemically distributed while phage type 193 is becoming an important phage type for Public Health and often associated with swine products (5).

*Corresponding Author. Mailing address: FIOCRUZ - Lab. de Referência Nacional de Cólera Enteroinfecções Bacterianas - Lab. de Enterobactérias Dep. de Bacteriologia - Av. Brasil, 4365, Manguinhos, Pavilhão Rocha Lima - $3^{\circ}$ andar - sala 322. CEP: $21040-360$ - Rio de Janeiro, RJ. E-mail: chrisspm@ioc.fiocruz.br 
The purpose of this retrospective study is to present the distribution of STM phage types in different sources in Brazil and the correspondent multidrug resistance profiles.

\section{MATERIALAND METHODS}

A total of 278 Salmonella Typhimurium (STM) strains isolated between 1999 and 2004 at the Laboratory of Enterobacteria, Oswaldo Cruz Institute, FIOCRUZ were phage typed using standard scheme (1) with 31 STM phage types, plus the phages 1,2,3,10 and 18. Phage suspensions and the interpretative guide were kindly provided by the International Reference Laboratory for Enteric Phage Typing, Health Protection Agency (HPA), Colindale, London, UK.

The STM strains have been isolated from animals (145), food for human consumption (77), food stuff (22), humans (10), environment (03), quality control (02) and other sources (19). They were analyzed for antimicrobial resistance to ampicillin 10 $\mu \mathrm{g}$ (AMP), chloramphenicol $30 \mu \mathrm{g}(\mathrm{CHL})$, tetracycline $30 \mu \mathrm{g}$ (TCY), cefoxitin $30 \mu \mathrm{g}$ (FOX), ceftriaxone $30 \mu \mathrm{g}$ (CRO), cyprofloxacin $5 \mu \mathrm{g}$ (CIP), gentamicin $10 \mu \mathrm{g}$ (GEN), imipenem 10 $\mu \mathrm{g}$ (IMP), nalidixic acid $30 \mu \mathrm{g}$ (NAL), trimethoprim/ sulfamethoxazole $1.25 \mu \mathrm{g} / 23.75 \mu \mathrm{g}$ (SXT), nitrofurantoin 300 $\mu \mathrm{g}$ (NIT) and cephalotin $30 \mu \mathrm{g}$ (CEP) using the disk diffusion method (CLSI, 2005). The interpretation of results were performed as described in the Clinical and Laboratory Standards Institute guidelines. Multidrug-resistance was defined as resistance to three or more classes of antimicrobials.

\section{RESULTS}

The STM strains were differentiated into 20 definitive phage types. DT 193 (64.3\%) was the predominant phage type, followed by DT $19(17.4 \%)$ and DT $18(4 \%)$. Other phage types were obtained in lower percentages: DT $27(3.24 \%)$, DT $10(2 \%)$, DT $42(1.08 \%)$, DT 89 (1.08\%), DT $44(0.72 \%)$, DT $168(0.72 \%)$, DT $13(0.36 \%)$, DT $22(0.36 \%)$, DT $26(0.36 \%)$, DT $28(0.36 \%)$, DT $29(0.36 \%)$, DT $45(0.36 \%)$, DT $53(0.36 \%)$, DT $55(0.36 \%)$, DT 168A (0.36\%), DT $149(0.36 \%)$ and DT 184 $(0.36 \%)$. Also, three phage types not considered definitive phages were isolated: U298 (0.72\%), U291 (0.36\%) and U294 $(0.36 \%)$ (Table 1$)$.

A total of 10 Salmonella Typhimurium isolates were isolated from humans during the period of the study. In 2002 the phage type DT193 was often isolated. In 2003, DT 19 (10\%), DT 13 (10\%) and DT $27(10 \%)$ were also identified. Among them only DT 193 isolated in 2002 showed multidrug resistance for more than three antimicrobial classes (Table 2).

The distribution of STM strains according to different sources from 1999 to 2004 showed that Salmonella Typhimurium strains were most common in materials from animal origin (43.4\%) and food for human consumption (32.7\%)(Table 3). Among the
Table 1. Salmonella Typhimurium phage types isolated from different sources over the period of 1999 to 2004 at LABENT/ IOC/FIOCRUZ, Brazil.

\begin{tabular}{cccc}
\hline Phage Type & $\mathrm{N}(\%)$ & Phage Type & $\mathrm{N}(\%)$ \\
\hline DT 193 & $178(64.3)$ & DT 26 & $01(0.36)$ \\
DT19 & $48(17.4)$ & DT 28 & $01(0.36)$ \\
DT 18 & $11(4)$ & DT 29 & $01(0.36)$ \\
DT27 & $09(3.24)$ & DT 45 & $01(0.36)$ \\
DT10 & $06(2)$ & DT 53 & $01(0.36)$ \\
DT 42 & $03(1.08)$ & DT 55 & $01(0.36)$ \\
DT 89 & $03(1.08)$ & DT149 & $01(0.36)$ \\
DT 44 & $02(0.72)$ & DT 168A & $01(0.36)$ \\
DT168 & $02(0.72)$ & DT 184 & $01(0.36)$ \\
U 298 & $02(0.72)$ & U 291 & $01(0.36)$ \\
DT 13 & $01(0.36)$ & U 294 & $01(0.36)$ \\
DT 22 & $01(0.36)$ & & \\
\hline
\end{tabular}

$\mathrm{N}=$ Number of Salmonella Typhimurium strains, \%=Percentage from the total Salmonella Typhimurium strains (278).

Table 2. Salmonella Typhimurium phage types isolated from humans over the period of 1999 to 2004 at LABENT/IOC/ FIOCRUZ, Brazil, and their antimicrobial profile.

\begin{tabular}{cccc}
\hline Year & Phage Type & N & Antimicrobial Profile \\
\hline 1999 & DT 193 & 2 & TCY \\
& & & TCY, SXT \\
2002 & DT 193 & 3 & $\begin{array}{c}\text { CHL, TCY, SXT, NIT } \\
\text { CHL, TCY, NAL, SXT, NIT } \\
\text { AMP, CHL, TCY, CEP, NAL, } \\
\end{array}$ \\
& & & SXT, NIT \\
2003 & DT193 & 2 & TCY \\
& & & AMP, TCY \\
& DT 13 & 1 & TCY \\
& DT 19 & 1 & - \\
& DT 27 & 1 & - \\
\hline
\end{tabular}

$\mathrm{AMP}=$ Ampicilin, $\mathrm{CEP}=$ Cephalotin, $\mathrm{CHL}=$ Chloramphenicol, $\mathrm{NAL}=$ Nalidixic Acid, NIT=Nitrofurantoin; SXT=Trimethoprim/ Sulfamethoxazole, TCY=Tetracycline.

278 STM analyzed for antimicrobial resistance, DT 193 (58\%), DT $19(16 \%)$ and DT18 (4\%) STM isolates showed characteristic to multiple antibiotic resistance in more than three antimicrobial classes but no resistance to third generation cephalosporin or cyprofloxacin (Tables 4 and 5). It was also observed that 14 different phage types non-DT 193 and 01 strain DT 19 were sensitive for the drugs tested (Table 6). 
Table 3. Distribution of Salmonella Typhimurium (STM) isolated according to their sources during the period of 1999 to 2004 at LABENT/IOC/FIOCRUZ, Brazil.

\begin{tabular}{|c|c|c|c|c|c|c|c|c|}
\hline \multirow{3}{*}{ Year } & \multicolumn{7}{|c|}{ Sources } & \multirow[b]{2}{*}{ Total } \\
\hline & AN & $\mathrm{EN}$ & $\mathrm{FH}$ & $\mathrm{HU}$ & FS & $\mathrm{QC}$ & US & \\
\hline & $\mathrm{N}(\%)$ & $\mathrm{N}(\%)$ & $\mathrm{N}(\%)$ & $\mathrm{N}(\%)$ & $\mathrm{N}(\%)$ & $\mathrm{N}(\%)$ & $\mathrm{N}(\%)$ & $\mathrm{N}(\%)$ \\
\hline 1999 & $14(5)$ & $03(1)$ & $21(7.6)$ & $02(0.7)$ & $12(4.3)$ & - & $01(0.4)$ & $53(19)$ \\
\hline 2000 & $12(4.3)$ & - & $03(1)$ & - & $04(1.4)$ & - & $02(0.7)$ & $21(7.6)$ \\
\hline 2001 & $09(3.2)$ & $01(0,4)$ & $01(0.4)$ & - & $02(0.7)$ & - & - & $13(4.7)$ \\
\hline 2002 & $05(1.7)$ & - & $28(10)$ & $03(1)$ & $02(0.7)$ & $01(0.4)$ & - & 39 (14) \\
\hline 2003 & $110(39.5)$ & - & $16(5.8)$ & $05(1.7)$ & $01(0.4)$ & - & - & $132(47.5)$ \\
\hline 2004 & - & - & $08(2.8)$ & - & $01(0.4)$ & - & $11(3.9)$ & $20(7.2)$ \\
\hline Total & $150(54)$ & 04 (1.4) & $77(27.6)$ & $10(3.6)$ & $22(8)$ & $01(0.4)$ & $14(5)$ & 278 \\
\hline
\end{tabular}

$\mathrm{N}=$ Number of STM strains, \%= Percentage of STM strains over the total (278), AN= Animal source, EN= Environmental source, FH= Food for human consumption source, $\mathrm{HU}=$ Human source, $\mathrm{FS}=$ Food stuff, QC= Quality Control source, US= unrelated source.

Table 4. Distribution of antimicrobial resistance profiles of S.Typhimurium DT 193 isolated from 1999 to 2004 at LABENT/ IOC/FIOCRUZ.

\begin{tabular}{|c|c|c|c|}
\hline Year & Source & $\mathrm{N}$ & Antimicrobial Resistance Profile \\
\hline \multirow{3}{*}{1999} & $\mathrm{FH}$ & 2 & $\begin{array}{l}\text { CHL, TCY, NIT } \\
\text { CHL, TCY,SXT }\end{array}$ \\
\hline & AN & 4 & $\begin{array}{l}\text { AMP, CHL, TCY } \\
\text { CHL, TCY, SXT } \\
\text { TCY, NIT, GEN } \\
\text { TCY, SXT, NIT }\end{array}$ \\
\hline & $\mathrm{FS}$ & 3 & $\begin{array}{l}\text { TCY, NIT, GEN } \\
\text { CHL, TCY, NIT, GEN } \\
\text { TCY, SXT, NIT, GEN }\end{array}$ \\
\hline \multirow{3}{*}{2000} & $\mathrm{FH}$ & 1 & AMP, CHL, TCY, GEN \\
\hline & $\mathrm{AL}$ & 7 & $\begin{array}{l}\text { AMP, TCY, GEN } \\
\text { AMP, CHL, TCY } \\
\text { CHL, TCY, NAL } \\
\text { AMP, CHL, TCY, SXT } \\
\text { AMP, TCY, NAL, NIT } \\
\text { CHL, TCY, SXT, NIT } \\
\text { CHL, TCY, NAL, SXT, NIT }\end{array}$ \\
\hline & US & 2 & $\begin{array}{l}\text { TCY, NAL, NIT } \\
\text { CHL, TCY, SXT }\end{array}$ \\
\hline & $\mathrm{EN}$ & 2 & $\begin{array}{l}\text { AMP, TCY, GEN } \\
\text { CHL, TCY, SXT }\end{array}$ \\
\hline 2001 & $\mathrm{AN}$ & 6 & $\begin{array}{l}\text { TCY, NAL, NIT } \\
\text { AMP, TCY, NAL, NIT } \\
\text { CHL, TCY, SXT, NIT } \\
\text { AMP, CHL, TCY, GEN, NIT }\end{array}$ \\
\hline
\end{tabular}

AMP, TCY, NAL, SXT, NIT AMP, CHL, TCY, NAL, SXT, NIT

\begin{tabular}{lll}
\multirow{2}{*}{ FS } & 2 & TCY, NAL, NIT \\
& & AMP, CHL, TCY, SXT \\
\hline & & TCY, NAL, NIT \\
& AMP, TCY, NIT \\
& CHL, TCY, NIT \\
& & TCY, GEN, NAL \\
& & TCY, NAL, SXT \\
& & CHL, TCY, NAL, NIT \\
& & CHL, TCY, SXT, NIT \\
& & AMP CHL, TCY, GEN, NIT
\end{tabular}

$\begin{array}{llll} & & & \text { TCY,NAL, NIT } \\ 2002 & \text { AN } & 4 & \text { AMP, TCY, NIT } \\ & & \text { AMP, CHL, TCY, NIT } \\ & & \text { AMP, CHL, TCY, CEP, NIT } \\ & \text { QC } & 1 & \text { CHL, TCY,NAL } \\ & & & \text { CHL, TCY, SXT, NIT }\end{array}$

HU 3 CHL, TCY, NAL, SXT, NIT AMP, CHL, TCY, CEP,NAL, SXT, NIT CHL, TCY, NAL, SXT, NIT

FS 2 AMP, CHL, TCY, CEP, GEN, NAL, SXT, NIT

CHL, TCY, NAL

TCY, NAL, NIT

CHL, TCY, GEN

CHL, TCY, NIT

TCY, GEN, NAL

AMP, TCY, NIT

CHL, TCY, SXT, NIT 
$\mathrm{AL}$

13

AMP, CHL, TCY, GEN
CHL, TCY, NAL, SXT
AMP, CHL, TCY, NAL, NIT
CHL, TCY, CEP, NAL, SXT
CHL TCY, GEN, NAL, SXT
CHL, TCY, GEN, NAL
SXT, NIT

2003
CHL, TCY, GEN

CHL, TCY, NAL

CHL, SXT, NIT

AMP, TCY, NIT

AN 10 CHL, TCY,GEN, NAL

AMP, CHL, TCY, NIT

AMP, CHL, TCY, GEN

TCY, GEN, NAL, SXT, NIT

\begin{tabular}{|c|c|c|c|}
\hline & & & $\begin{array}{l}\text { AMP, CHL, TCY, GEN, NIT } \\
\text { AMP, CHL, TCY, NAL, SXT, NIT }\end{array}$ \\
\hline & FS & 1 & AMP, CHL, TCY, NAL, SXT \\
\hline & US & 1 & TCY,NAL, NIT \\
\hline \multirow[t]{2}{*}{2004} & US & 5 & $\begin{array}{l}\text { TCY, SXT, NIT } \\
\text { TCY, NAL, SXT } \\
\text { TCY, NAL, SXT, NIT } \\
\text { AMP, TCY, CEP, CRO, SXT } \\
\text { AMP, CHL, TCY, CEP, CRO, NIT }\end{array}$ \\
\hline & $\mathrm{FH}$ & 1 & CHL, TCY,NIT \\
\hline
\end{tabular}

$\mathrm{N}=$ antimicrobial profile number $\mathrm{AMP}=\mathrm{Ampicilin}, \mathrm{CEP}=$ Cephalotin, $\mathrm{CHL}=$ Chloramphenicol, $\mathrm{CRO}=$ Ceftriaxone, $\mathrm{GEN}=$ Gentamicin, $\mathrm{NAL}$ $=$ Nalidixic Acid, NIT $=$ Nitrofurantoin, SXT $=$ Trimethoprim $/$ Sulfamethoxazole, $\mathrm{TCY}=$ Tetracycline.

Table 5. Salmonella Typhimurium non-DT 193 phage types isolated from 1999 to 2004 from different sources and classified according to their antimicrobial resistance profile.

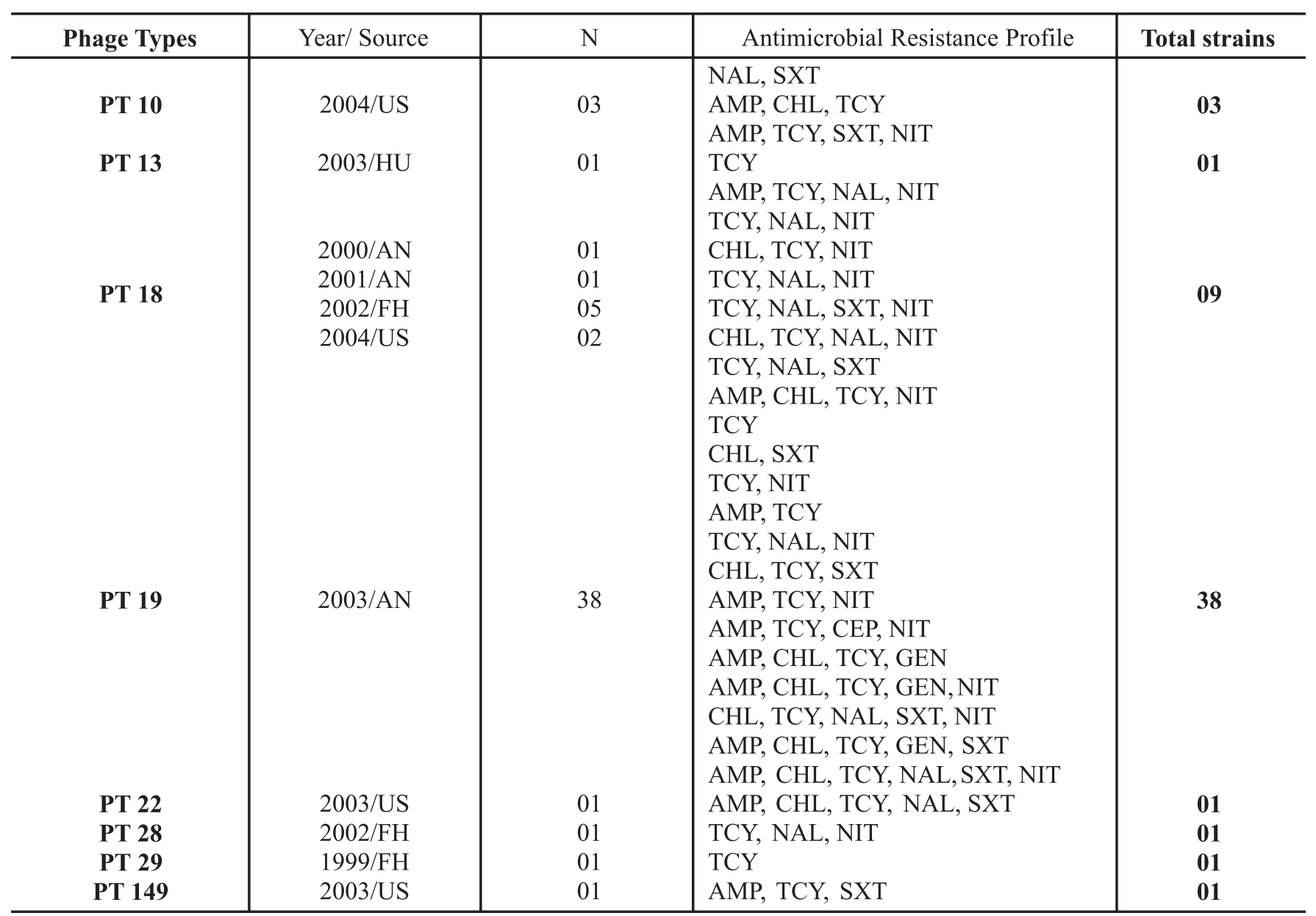

$\mathrm{N}=$ Number of Salmonella Typhimurium isolated according to year and source, AN= Animal source, FH= Food for human consumption source, $\mathrm{HU}=$ Human source, $\mathrm{US}=$ Unrelated source; $\mathrm{AMP}=\mathrm{Ampicilin}, \mathrm{CEP}=$ Cephalotin, $\mathrm{CHL}=\mathrm{Ch}$ loramphenicol, $\mathrm{GEN}=\mathrm{Gentamicin}, \mathrm{NAL}=\mathrm{Nalidixic}$ Acid, NIT=Nitrofurantoin; SXT=Trimethoprim/Sulfamethoxazole, TCY=Tetracycline. 
Table 6. Salmonella Typhimurium non-DT193 phage types isolated from 1999 to 2004 and sensitive to all drugs tested.

\begin{tabular}{cc}
\hline Phage Types & $\mathrm{N}(\%)$ \\
\hline DT 19 & $1(4.3)$ \\
U291 & $1(4.3)$ \\
DT 26 & $1(4.3)$ \\
DT 27 & $1(4.3)$ \\
DT45 & $1(4.3)$ \\
U294 & $1(4.3)$ \\
DT 168A & $1(4.3)$ \\
DT 53 & $1(4.3)$ \\
DT 184 & $1(4.3)$ \\
DT 168 & $2(8.7)$ \\
U 298 & $2(8.7)$ \\
DT 44 & $2(8.7)$ \\
DT 55 & $2(8.7)$ \\
DT 42 & $3(13)$ \\
DT 89 & $3(13)$ \\
\hline Total & $\mathbf{2 3 ( 1 0 0 )}$ \\
\hline
\end{tabular}

$\mathrm{N}=$ Number of Salmonella Typhimurium strains, \%=Percentage of Salmonella Typhimurium strains over the total (23).

\section{DISCUSSION}

In the present study the prevalence of Salmonella Typhimurium isolated from animals and food for human consumption associated with multidrug resistance in Brazil has determined the need to monitor the spread of this microorganism (8).

Phage typing distinguished serotype Typhimurium variants in 23 different phage types. Among them a high prevalence of resistant strains was especially detected in phage type DT 193, which can be corroborated with studies that demonstrate the emergence of this phage type associated with swine, food for human consumption and antimicrobial resistance (7).

In Brazil, Salmonella Typhimurium phage types isolated from humans appear to have a geographical distribution pattern. The main phage type isolated in areas such as Rio de Janeiro was DT 193, whereas DT 19, DT 41, DT 97, DT 105, DT 120 and DT 193 were isolated in Salvador city. In both cases, all strains were isolated from children hospitalized with enteric processes (2).

In the present investigation it was observed that DT 193 was the most prevalent phage type isolated from humans associated with multidrug resistance to more than three antimicrobials. Other phage types were also isolated, such as DT 13 resistant only to tetracycline, and DT 19 and DT 27 sensitive for all drugs tested.
Although our findings did not indicate the presence of DT 104 , considered an emergent phage type, this retrospective study allows isolating Salmonella phage types DT 193 and non 193 associated with a high level of multidrug resistance. Moreover, the wide variety of phage types isolated from different sources, including human source, shows the rapid spread of Salmonella Typhimurium circulating in Brazil from 1999 to 2004 (4).

Taking into account the data presented in this paper, it is important that a prudent use of antimicrobials in veterinary field, agriculture and treatment patients should be encouraged, in order to contribute in minimizing the emergence of multidrug resistance. Furthermore, the surveillance of Salmonella Typhimurium strains based on phage typing is useful for detecting outbreaks, identifying sources of infection and implementing prevention and control measures.

\section{RESUMO \\ Fagotipagem e Perfil de multirresistência antimicrobiana em $S$. Typhimurium isoladas de diferentes fontes no Brasil de 1999 a 2004}

Salmonella Typhimurium é considerada uma das principais bactérias causadoras de salmonelose nos animais e no homem em todo o mundo. No Brasil, Salmonella Typhimurium é um dos mais prevalentes sorovares isolados de alimentos para consumo humano. $\mathrm{O}$ uso indiscriminado de antibióticos em produtos agrícolas e no tratamento de pacientes humanos tem contribuído para aumentar a multirresistência desse sorovar a diversos antimicrobianos. No presente estudo, 278 cepas de STM foram selecionadas de diferentes fontes e regiões do Brasil, no período de 1999 a 2004 e realizadas a fagotipagem e análise do perfil de resistência antimicrobiana no Laboratório de Enterobactérias, Instituto Oswaldo Cruz, FIOCRUZ. Os principais fagotipos isolados foram DT 193 (64,3\%), DT 19 $(17,4 \%)$ e DT $18(4 \%)$. Os fagotipos DT $10(2 \%)$, DT $27(3,24 \%)$, DT $13(0,36 \%)$, DT $22(0,36 \%)$, DT $28(0,36 \%)$, DT $29(0,36 \%)$ e DT $149(0,36 \%)$ foram isolados em menores percentuais. Um total de 54\% das cepas de STM foi resistente a três ou mais classes de antimicrobianos e não foi observada resistência a cefalosporinas de terceira geração ou ciprofloxacina. Esses resultados indicam os principais lisotipos de Salmonella Typhimurium circulantes entre os animais, alimentos de consumo humano e seres humanos no Brasil, bem como o aumento da multirresistência antimicrobiana. O monitoramento de cepas de Salmonella Typhimurium baseado na fagotipagem e no padrão de resistência antimicrobiana são ferramentas úteis para detectar surtos, identificar a fonte de infecção e implementar programas de prevenção e controle de salmonelose.

Palavras-chave: Salmonella Typhimurium, Fagotipagem, Multirressistência 


\section{REFERENCES}

1. Anderson, E.S.; Ward, L.R.; Saxe, M.I.; de Sá, J.D.H. (1977). Bacteriophage-typing designations of Salmonella typhimurium.J. Hyg., 78(2), 297-300.

2. Asensi, M.D.; Costa, A.P.; Reis, E.M.F.; Hofer, E. (1995). Lysotypes and plasmidial profiles of Salmonella serovar Typhimurium isolated from children with enteric process in the cities of Rio de Janeiro, RJ, and Salvador, BA-Brazil. Rev Inst Med Trop São Paulo, 37(4), 297302.

3. CLSI. (2005). Clinical and Laboratories Standards Institute. Performance standards for antimicrobial susceptibility testing fifteenth informational supplement M100-S15, Wayne (PA), CLSI.

4. Ghilardi, A.C.R.; Tavechio, A.T.; Fernandes, S.A. (2006). Antimicrobial susceptibility, phage types, and pulsetypes of Salmonella Typhimurium, in São Paulo, Brazil. Mem. Inst. Oswaldo Cruz, 101(3), 281-286.
5. Liebana, E.; Garcia-Migura, L.; Clouting, C.; Clifton-Hadley, F.A.; Lindsay, E.; Threlfall, E.J.; McDowell, S.W.; Davies, R.H. (2002). Multiple genetic typing of Salmonella enterica serotype typhimurium isolates of different phage types (DT104, U302, DT204b, and DT49) from animals and humans in England, Wales, and Northern Ireland. J. Clin. Microbiol., 40(12), 4450-4456.

6. Popoff, M.Y. (2001). Antigenic formulas of Salmonella serovars. WHO Collaborating Centre for Reference Research on Salmonella, Institut Pasteur, Paris.

7. Tavechio, A.T.; Ghilardi, A.C.; Peresi, J.; Fuzihara, T.O.; Yoramine, E.K.; Jakabi, M.; Fernandes, S.A. (2002). Salmonella serotypes isolated from nonhuman sources in Sao Paulo, Brazil, from 1996 through 2000. J. Food Prot., 65(6), 1041-1044.

8. Weill, F.X.; Guesnier, F.; Guibert, V.; Timinouni, M.; Demartin, M.; Polomack, L.; Grimont, P.A. (2006). Multidrug Resistance in Salmonella enterica Serotype Typhimurium from Humans in France (1993 to 2003) J. Clin Microbiol., 44(3), 700-708. 